\title{
Hostility, facial configuration, and bilateral asymmetry on galvanic skin response
}

\author{
MATTHEW L. HERRIDGE, DAVID W. HARRISON, and HEATH A. DEMAREE \\ Virginia Polytechnic Institute and State University, Blacksburg, Virginia
}

\begin{abstract}
The effects of hostility on bilateral measures of skin conductance during affective facial configurations were investigated. Highly hostile and non-hostile men were instructed in making facial configurations that were identified by raters as happy, angry, or neutral. Subjects were asked to make the set of facial configurations twice with unstructured baselines taken prior to each expression. Significant increases in skin conductance were found from baseline across all three facial configurations. Skin conductance varied as a function of affect, decreasing from angry to happy to neutral configurations. A three-way group $\times$ hand $\times$ block interaction was found. The high-hostile group showed persistence in the elevation of conductance at the left hand through Block 2 . In contrast, reduced skin conductance at the left hand was found in the low-hostile group, along with habituation at the left hand across blocks. The possibility of altered right cerebral systems in high-hostile individuals is discussed.
\end{abstract}

A link between hostility and the body's physiological response to it has long been an area of study within the varied disciplines of psychological research. The physiological manifestations of anger were recorded by Wolff (1950), who examined a patient who had a gastric fistula that allowed his gastric mucosa to be viewed. When the patient felt anger, hostility, or resentment, the mucosa became red in color, engorged with blood, and produced more secretions. In 1953, Ax noted that anger was associated with increased blood pressure and heightened electromyogram and skin conductance responses.

Both anger and altered autonomic reactivity may correlate with right-hemisphere activation. Demaree and Harrison (in press-c) found right cerebral activation and cardiovascular reactivity to cold-pressor pain in highhostile men men using a cerebral laterality task. Nonhostile men responded with increased left cerebral activation and cardiovascular stability. Heilman, Bowers, and Valenstein (1993) theorized that patients with righthemisphere damage may have an inadequate arousal level due to problems associated with the brainstem and thalamic activating systems. Heilman et al. then found hypoarousal and hyporeactivity using galvanic skin response (GSR) among right-hemisphere-damaged patients (many with posterior lesions). Further evidence of a unilateral neglect in these patients supported the hypothesis of an attention or arousal defect.

Other evidence involves expressive defects associated with left-hemisphere damage. Nonfluent aphasics can become very fluent when using expletives. Even aphasic patients with agraphia were able to write emotional

Correspondence should be addressed to D. W. Harrison, Department of Psychology, Virginia Polytechnic Institute and State University, Blacksburg, VA 24061.

-Accepted by previous editor, Paul E. Gold words better than nonemotional words. Thus, the righthemisphere dominance in emotional expression seems to become more evident in unilaterally brain-damaged individuals.

It can also be hypothesized that dysfunction of the anterior right hemisphere may result in autonomic reactivity as well as a more prevalent hostile expression. The basal forebrain is thought to interact in a regulatory fashion with the amygdaloid bodies of the temporal lobe (e.g., Butters, Pandya, Sanders, \& Dye, 1971). Altered arousal/activation of these regions may act in a reciprocal fashion on both anger levels and autonomic reactivity. More specifically, afferent emotional stimuli such as visual, auditory, and kinesthetic (from the regulation of facial expression) cues may differentially impact hostile individuals, with the potential for relatively heightened activity within the right cerebral systems. Within the visual modality, some support for this hypothesis was found using tachistoscopic presentations of facial affect stimuli (Harrison, Gorelzcenko, \& Cook, 1990).

Harrison, Gorelczenko, and Cook (1990) found that high-hostile individuals were more likely to identify neutral faces as angry than were low hostiles. This was suggestive of a negative affective bias among these individuals. Interestingly, this effect was lateralized with heightened negative affect bias resulting from the presentation of neutral faces to the right cerebrum (left visual field). Thus, high hostiles potentially have a right cerebral predisposition including a negative affective bias and altered autonomic nervous system regulation.

Given previous research linking right cerebral arousal to both hostility and physiological regulation, it is not surprising that high, relative to low, hostiles have evidenced heightened physiological lability to stress. For example, Suarez and Williams (1989) found that high-hostile men experienced greater heart rate, blood pressure, and blood flow increases to an anagram task accompa- 
nied by harrassment. Similarly, Siegman, Anderson, Herbst, and Boyle (1992) found a significant and positive correlation between hostility level and blood pressure increases to a serial subtraction task. Perhaps most pertinent to this research, Demaree and Harrison (in press-c) used a dichotic listening paradigm and found that highrelative to low-hostile men showed both greater increases of heart rate and right cerebral arousal to a frequently used stressor, the cold pressor test.

The present experiment seeks to investigate facial configuration as an afferent affective stimulus that may differentially alter autonomic responses as a function of hostility. The expression of voluntary emotional facial configurations was used to induce corresponding autonomic reactions, measured by skin conductance. It is now commonly accepted that the expression of negatively valenced emotion (e.g., anger) is lateralized generally to the right hemisphere (see Demaree \& Harrison, in pressb), whereas the expression of positively valenced emotion (e.g., happiness) is controlled more globally by both hemispheres (Silberman \& Weingartner, 1986). For example, in relation to hostility, Edlund, Swann, and Clothier (1987) found heightened right temporal arousal in patients exhibiting intermittent episodes of excessive anger expression. Similarly, Heath (1982) and Demaree and Harrison (in press-a) found that heightened right temporal lobe arousal significantly corresponded with subjects' aggressive behavior.

Two studies using similar protocols elicited spontaneous negative and positive facial expression via presentation of short film clips (Davidson, Ekman, Saron, \& Senulis, 1990; Ekman, Davidson, \& Friesen, 1990). These researchers found that the expression of negative and positive facial valences was associated with right- and leftcerebral arousal. Positive emotional expression has been somewhat more difficult to lateralize, however. Research of brain lesions reducing happiness, or increasing depression, clearly exemplifies this notion.

Robinson, Kubos, Starr, Rao, and Price (1983) found that nearly two thirds of their subjects with left anterior lesions evidenced major depression. Other research has shown a positive relationship between the proximity of the lesion to the left frontal pole and the severity of the depression (Robinson, Kubos, Starr, Rao, \& Price 1983; Starkstein, Robinson, \& Price, 1987). Conversely, righthemisphere strokes have also been associated with depression. For example, both Folstein, Maiberger, and $\mathrm{McHugh}$ (1977) and Grafman, Vance, Weingartner, Salazar, and Amin (1986) found that their right-hemisphere stroke patients displayed depressive symptomatology significantly more than did their left-hemisphere stroke patients.

The neural systems theory of emotion and the theory of altered autonomic functioning in high hostiles were tested by having subjects coded as high hostile and low hostile make facial configurations identified by raters as happy, angry, and neutral facial affects while bilateral measures of skin conductance were taken.

\section{Hypotheses}

The hypotheses were as follows: (1) Higher skin conductance levels for the facial configurations than for the baseline would be found; (2) more reactivity would be noted for angry faces, followed by happy faces, and then neutral faces; (3) the left hand would show higher conductance levels than the right; (4) the high-hostile group would show higher conductance levels than the lowhostile group; and (5) a three-way interaction of group, hand, and affective facial configuration was predicted.

\section{METHOD}

\section{Subjects}

Twenty-six men, screened for right lateral preference, were selected from an undergraduate subject sample of 102 students on the basis of their scores on the Cook-Medley Hostility Scale (CMHO; Cook \& Medley, 1954) and the Framingham Type A Scale (Haynes, Levine, Scotch, \& Kannel, 1978). Subjects were placed into one of two groups: high-hostile individuals $(n=13)$, or low-hostile individuals ( $n=13$ ). Individuals (identified by a history questionnaire) who had a known neurological problem, who had a medical illness, or who had experienced significant head trauma were excluded The Beck Depression Inventory (BDI) and the State-Trait Anxiety Scale (STAS) were also administered in order to examine characteristics of the subject pool. Subjects also read and signed an informed consent form.

Handedness and hostility classification. A 13-item, behaviorally validated questionnaire (Coran, Porac, \& Duncan, 1979) assessing four types of lateral preference (hand, foot, eye, and ear) was administered. The self-report items are scored as +1 for right, -1 for left, and 0 for both left or right lateral preference. The questionnaire was developed from other laterality inventories and tests of hand preference. Criterion for acceptance and right lateral preference was a score of +6 or above $(\max =+13)$ on the questionnaire.

The hostility classifications were made using the $\mathrm{CMHO}$ and the Framingham Type A Scale (FTAS). The FTAS contains 10 items that are intended to assess feelings of time urgency, competitiveness, and "hard-driving" behaviors. It has been shown to correlate significantly with the Jenkins Activity Survey (Jenkins, Rosenman, \& Friedman, 1967) and the structured interview (Haynes, Feinleib, \& Kannel, 1980) in Type A classification. Many studies of hostility have used the MMPI Hostility scale devised by Cook and Medley in 1954. This 50-item questionnaire has been shown to be related to coronary artery disease (CAD) and the Type A Behavior Pattern (TABP). Individuals who score higher on the CMHO experience anger at higher intensities and frequencies than do low scorers.

Subjects were classified as high hostile who scored +6 or above on their FTAS and concurrent scores of 29 or above on the CMHO. Individuals were classified as low hostile who scored 5 or below on the FTAS and 20 or lower on the CMHO. Using two measures to assess hostility has been reported to improve homogeneity and validity of appropriate subtypes and has been used before (Muranka et al., 1988, Williams et al., 1982).

\section{Apparatus}

Physiological. To help assess differential cerebral arousal, a Grass Model 7D polygraph unit with two Model 7P1 DC preamplifiers was used to collect the skin conductance data across each measurement period. Beckman electrodes and Spectra 360 electrode gel were placed on opposite sides (hypothenar and thenar eminences) of the subject's lower palmar surface of each hand. Amplifiers and electrode leads were fully counterbalanced across subjects. The experimental chamber contained a large cushioned chair 
facing a one-way observation window surrounded equidistantly on each side and the front and back by a white curtain. The experimenter was seated in a separate sound-attenuated room with the polygraph unit. Subjects were monitored through the one-way observation window and a small hole in the curtain. Electrode attachment cables were connected to the polygraph unit via a small opening in the wall.

Video. A Panasonic WV-CD20 CCTV camera with a Panasonic WV-LA8B lens was placed behind the one-way observation window to record the subject's facial configurations throughout the experiment.

\section{Procedure}

The CMHO, FTAS, BDI, STAS, and lateral preference and history questionnaires were administered to the subject sample pool. Twenty-nine subjects were selected using the criteria mentioned above. Three declined to participate further, leaving a total of 26 subjects, with 13 in the high-hostile group and 13 in the low-hostile group. After reading and signing the informed consent forms, the subject was seated in the recliner's upright position. Electrodes were placed and the subject was informed that he would be asked "to move certain facial muscles." The experimenter then stated, "It is very important for you to follow precisely the directions I will give you. You may feel uncomfortable at times, but please try to maintain the facial configurations described to you. Let's practice a few." At this time, the experimenter returned to the other room and began to instruct the subject in making the three facial configurations-happy, angry, and neutral. At no point in the experiment were any of the facial configurations referred to as "happy," "angry," or "neutral," however. After successful completion of the facial configurations in the practice phase, the subject was told to relax, and a 3-minute baseline of skin conductance was taken. Following the baseline, in which subject's eyes remained open, the experimenter instructed the subject on how to make the first facial configuration again. The experimenter then said "Begin" and a 15 -sec measurement was made. At the end of this period, the subject was told to discontinue the configuration and relax. No formal relaxation procedures were used. The subject was monitored to ensure that emotional facial configurations were not being made during the baseline period. Another 3-min baseline was then taken. Following this, the experimenter instructed the subject in making the next facial configuration, and it was held for $15 \mathrm{sec}$. The subject was again asked to relax for a period of $3 \mathrm{~min}$. Following this, the experimenter instructed the subject in making the third facial configuration, and it was held for $15 \mathrm{sec}$. After another 3 -min baseline, the procedure was repeated following the same pattern as before. Thus, two recording blocks for each facial configuration were attained. Following the last facial configuration trial, the electrodes were removed and the subject was debriefed. The order of facial configurations was counterbalanced across subjects.

Validation of facial configurations. Each subject was viewed in a retrospective playback of the videotape by two associate researchers trained by the primary researcher. They then rated the intensity of each facial configuration as well as the subject's success at meeting the necessary criteria for the configuration and maintaining it for the appropriate time period (see Results).

Facial configuration instructions. The experimenter instructed the subjects on the angry facial configuration as follows: "Now I want you to pull your eyebrows down and together, raise your upper eyelid, and push your lower lip up." The experimenter instructed the subjects on the standard or control face as follows: "I want you to close your eyes and puff your cheeks out gently." The experimenter gave the following instructions for the happy facial configuration: "Now I want you to lift your cheeks up, pull them back, and open your mouth slightly." These instructions were adopted from previous research by Ekman, Levenson, and Friesen (1983).

\section{RESULTS}

Skin conductance data were analyzed with a five-factor mixed design repeated measures analysis of variance (ANOVA) with the fixed effect of group (high and low hostility) and the repeated factors of facial affect (happy, neutral, and angry), hand (left and right hands), block (first and second), and trial (baseline and test). Significance levels were computed with conservative degrees of freedom (Greenhouse \& Geisser, 1959). Post hoc comparisons were performed with Tukey's Honestly Significant Difference procedure (HSD; $\alpha=.05$ ).

Manipulation checks revealed that only 2 subjects had difficulty making the facial configurations (scoring an average of 2 or below on a 5-point Likert scale of facial configuration success) and that their difficulty was limited to the affective faces. Also, the groups were equal on the scale in the emotional intensity of their facial configurations as recorded by raters (Rater 1: high-hostile $M=$ $3.52, S D=1.41$, low-hostile $M=3.33, S D=1.29$; Rater 2: high-hostile $M=3.44, S D=1.15$, low-hostile $M=3.26, S D=1.13$ ).

The high-hostile group had a mean CMHO score of 32.9 $(S D=3.6)$, and the low-hostile group a mean of 16.2 $(S D=2.9)$. Scores ranged from 29 to 40 in the highhostile group and from 11 to 20 in the low-hostile group. Mean scores and standard deviations for the other instruments were as follows: high-hostile FTAS $=7.54, S D=$ $1.08 ; \mathrm{BDI}=11.31, S D=4.98 ;$ STAS-State $=37.85$, $S D=7.23$; STAS-Trait $=46.54, S D=10.63 \cdot$ low-hostile FTAS $=3.62, S D=1.33$; BDI $=3.15, S D=2.99$; STASState $=31.23, S D=7.5 ;$ STAS-Trait $=32.69, S D=$ 8.25. Correlation analysis of the initial pool of subjects showed significant relationships among the CMHO, FTAS, BDI, and STAS.

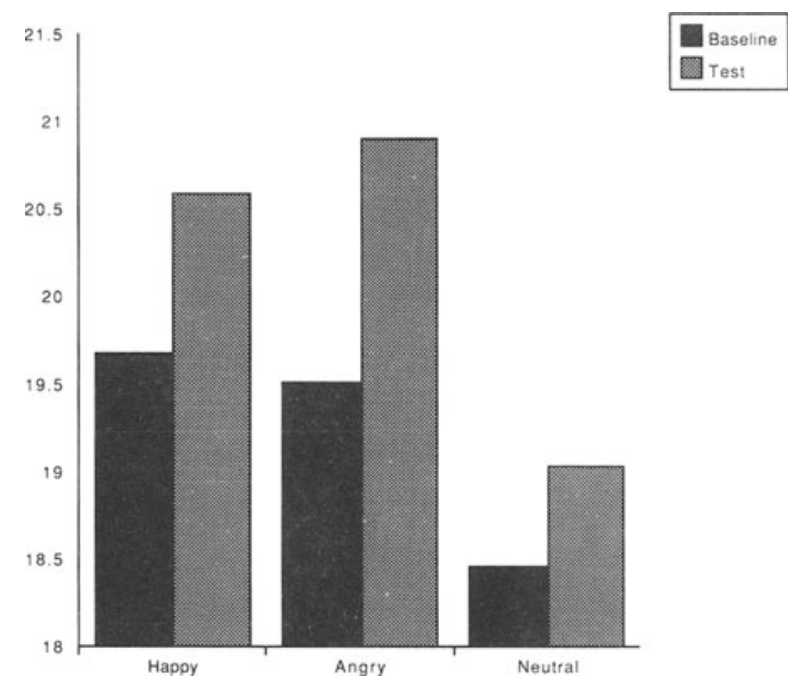

Figure 1. Skin conductance measured in micromhos as a function of affect and trial. 


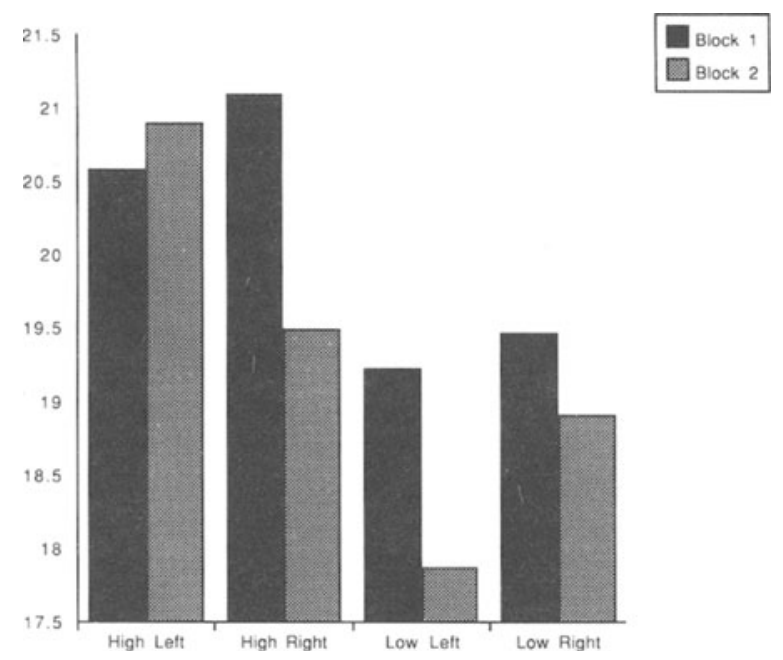

Figure 2. Skin conductance measured in micromhos as a function of group, hand, and block.

Analysis of the skin conductance data demonstrated that Hypothesis 1 was confirmed by a main effect of trial. Significantly higher skin conductance levels were noted during the facial configurations than during the baseline measures $[F(1,24)=16.61, p<.0004]$. The affect $\times$ trial interaction was also significant and confirmed Hypothesis 2 (however, see Discussion). More reactivity (change from baseline to facial configuration) was seen for angry faces, followed by happy and then neutral $[F(2,48)=$ $8.96, p<.0005$; see Figure 1). Post hoc comparisons indicated that conductance varied within each facial configuration from baseline to test. However, among the angry and happy facial configurations, conductance was not significantly different at baseline or test. Conductance during both the angry and happy configurations was significantly different from neutral both at baseline and test. It should also be noted that in a separate test of only the happy and angry facial configurations, reliable differences in reactivity were found.

Hypothesis 3 received partial support only (see below). No significant interaction effect was noted for group and affect. Hypotheses 4 and 5 were not confirmed. The group $X$ hand interaction and group $X$ hand $X$ affect interaction were not significant. Another significant three-way interaction was found, however. The group $x$ hand $x$ block interaction $[F(1,24)=8.12, p<.0089]$ demonstrated a clear distinction among the groups. Moreover, group differences (Hypothesis 4) were confirmed by Block 2 with heightened skin conductance found at the left hand of the high-hostile group. Diametrically opposite effects of hand were found in the low-hostile group (Figure 2).

Post hoc comparisons indicated that during Block 1, both hands of the high-hostile group showed significantly more conductance than both hands of the lowhostile group. However, during Block 2, the right hand of the high-hostile group could only be differentiated from the left hand of the low-hostile group. Conductance at the left hand of the high-hostile group was now significantly beyond that of either hand of the low-hostile group, as was hypothesized. No other main effects or interactions were reliable using the corrected degrees of freedom. Figure 3 depicts a relationship which failed to meet these more stingent requirements $(p<.056)$.

\section{DISCUSSION}

The primary finding of the present experiment was that men with high levels of hostility show heightened autonomic responses at the left hand over time when making affective facial configurations. This finding was opposite to that of the low-hostile group, for whom lowered conductance was associated with the left hand. The results also indicate that happy, angry, and neutral affective facial configurations may differentially induce autonomic correlates of heightened skin conductance.

First, a significant three-way interaction of group, hand, and block was found. Autonomic responses at the right hand in the high-hostile group varied across blocks. Diametrically opposite results were found for the left hand in this group, which showed persistently high conductance values across blocks. Initially (Block 1), the left hand of the high-hostile group showed equivalent conductance to that measured at the right hand. The high-hostile group showed higher levels of conductance at both hands at this initial point in the session. However, by Block 2 , the right hand was approaching the conductance levels of the lowhostile group. Habituation seemed to have occurred in bilateral hand measures of the low-hostile group, whereas evidence of habituation was seen only in the right-hand measure of the high-hostile group. Measurement at the left hand of the high-hostile group showed persistence in high levels of skin conductance. This finding is sugges-

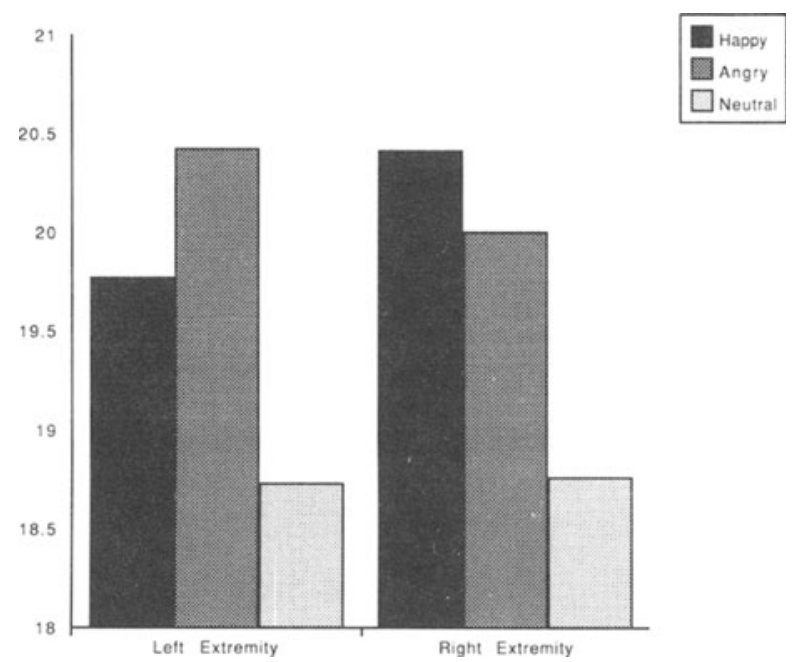

Figure 3. Skin conductance measured in micromhos as a function of the nonsignificant hand $\times$ affect interaction. 
tive of asymmetry in habituation and, specifically, slower habituation at the left hand.

An explanation involving altered right-hemisphere function in men with high levels of hostility seems appropriate for this phenomenon. Research indicates that inertia of affect (Cummings \& Mendez, 1984; Gainotti, 1972) and affective lability (Robinson, Parikh, Lipsey, Starkstein, \& Price, 1993) may result from right-hemisphere dysfunction. These individuals are likely to respond in an affectively inappropriate way to various cues. They are also likely to "perseverate" on negative emotional valences. In a recent case study (Demakis, Herridge, \& Harrison, 1994) on affective lability in a patient following a right-hemisphere cerebrovascular accident, a similar effect was noted. The patient showed heightened levels of skin conductance and reactivity to emotional cues accompanied by behavioral lability. Though this was an extreme case, dysfunction within this cerebrum may possibly produce similar behavior of smaller magnitude, such as that seen in the hostility disorders (e.g., Type A behavior pattern). The higher conductance level at the left hand of the high-hostile group is consistent with this hypothesis.

If high hostiles do perseverate autonomically after reacting to an emotional incident, a link to the literature on cardiovascular risk in this population may be indicated. This integration of the neuropsychological approach to affect disorders may offer researchers a novel way in which to view heightened levels of hostility and perhaps cardiovascular risk as well. These individuals may react more and maintain states of higher autonomic arousal for longer periods of time than do low hostiles because of altered right-hemisphere functioning. This may be triggered by emotional stimuli of either valence, or through altered appraisal of neutral stimuli as having a negative valence (Harrison et al., 1990).

Second, it was found that simply moving facial muscles into a configuration universally recognized as happy, angry, or neutral produced skin conductance. It was also found that the angry, happy, and neutral configurations produced skin conductance responses that varied in magnitude, respectively. As predicted, the angry facial configuration showed the highest reactivity, followed by the happy facial configuration, and then the neutral facial configuration. The angry configuration resulted in reactivity beyond that found for happy configurations. Both angry and happy configurations resulted in reactivity beyond that found for the neutral configuration.

Discussion of the latter finding (i.e., neutral affect) must be qualified because of baseline discrepancies among the affect types. The neutral baseline differed from the baseline values of the other affect valences. Exploration of the neutral baseline difference in the affect $\times$ trial interaction revealed that the neutral facial configuration occurred less often as the first or second facial configuration than did the happy or angry facial configurations (happy $=18$, angry $=19$, neutral $=15$ ). This is likely to have produced the disparity between the neutral base- line and the angry and happy baselines because habituation seems to have occurred across time in this experiment.

Baseline conductance levels dropped during the second block as the subjects habituated to the experimental situation. Thus, the more often a facial configuration occurred as the last of the three, the more likely it was to have a lower mean skin conductance value. Sturgis and Gramling (1988) have pointed out that a common problem in electrodermal measurement is that level changes may be of a relatively large magnitude compared with responses elicited by specific stimuli. However, the law of initial values (LIV; Wilder, 1931/1957), which suggests that the greatest reactivity of a dependent variable to an experimental manipulation most likely results when the baseline DV is lowest, suggests that this interaction is not confounded. Within-subjects measures, behaving in accordance with the LIV, would be predicted to show more of a response increment in conditions in which the initial values were lower (Furedy \& Scher, 1989). Thus, the neutral configuration would be expected to show greater increases than would the angry or happy configurations. This was not the case. The affective faces showed heightened responsivity (reactivity) over that of the neutral face.

Clear evidence for the neural systems theory of affect was not found. Positive and negative facial configurations were not reliably discriminated across hands. However, the hands did show a trend wherein different patterns of skin conductance were evident (see Figure 3 ). Neutral affect was more similar across hands than was happy or angry affect. Angry affect resulted in higher levels of conductance than did happy affect configurations at the left hand, whereas they were more similar on the right hand. This offers partial support for the utility of neural systems theory in the study of affective lateralization.

Skin conductance reactivity to the facial configurations may also show greater differentiation across hands and between groups with longer experimental sessions. This might help confirm experimental hypotheses regarding hand variations among facial configurations that were not found in this experiment.

The results, though, may have been limited by the nature of the facial affect task. Recent research has indicated that physiological effects may vary across types of happy facial configurations. Thus, the facial configuration should involve musculature that has been shown to elicit autonomic responses most similar to those produced by spontaneous and intense facial configurations. It is important to note that the present research did not elicit spontaneous expressions of a happy facial configuration previously associated with heightened left cerebral arousal (Davidson et al., 1990; Ekman et al., 1990). Such spontaneous, rather than involuntary, facial expression may improve future research in this domain.

Future research should focus on altered autonomic expression in individuals with high levels of hostility in response to emotional or nonemotional cues. Other types of autonomic responses, such as heart rate, blood pressure, and blood volume pulse may be appropriate because of 
their relevance to cardiovascular risk. This autonomic alteration (or dysfunction in terms of social interaction and cardiovascular risk) in high-hostile individuals, if confirmed, could help link the hostility/coronary artery disease literature with that of functional cerebral systems. The integration of literatures on cardiovascular risk and the neuropsychology of hostility (e.g., Demaree \& Harrison, in press $-b$, in press-c) is needed to open potentially vital future research avenues. Also, continued use of bilateral measurements is recommended in research using skin conductance as an expression of cerebral activity. Different results across hands are likely, especially in experiments using affective variables.

\section{REFERENCES}

Ax, A. F. (1953). The physiological differentiation between fear and anger in humans. Psychosomatic Medicine, 15, 433-442.

Butters, N., PANdya, D., Sanders, K., \& Dye, P. (1971). Behavioral deficits in monkeys after selective lesions within the middle third of sulcus principalis. Journal of Comparative \& Physiological Psychology, 76, 8-14.

Cook, W., \& MedLey, D. (1954). Proposed hostility and pharasaicvirtue scales for the MMPI. Journal of Applied Psychology, 238, 414-418.

Coran, S. P., Porac, C., \& Duncan, P. (1979). A behaviorally validated self-report inventory to assess four types of lateral preferences. Journal of Clinical Neuropsychology, 1, 55-64.

Cummings, J. L., \& MENDEZ, M. P. (1984). Secondary mania with focal cerebrovascular lesions. American Journal of Psychiatry, 141, 1084-1087.

Davidson, R. J., Ekman, P., Saron, C. D., \& Senulis, J. A. (1990). Approach-withdrawal and cerebral asymmetry: Emotional expression and brain physiology. Journal of Personality \& Social Psychology, 7, 201-207.

Demakis, G., Herridge, M. L., \& Harrison, D. W. (1994). Pathologic display of positive affect: A bilateral electrodermal case study. Neuropsychiatry, Neuropsychology, \& Behavioral Neurology, 7, 154-159.

Demaree, H. A., \& HarRison, D. W. (in press-a). Electroencephalography of hostility following closed head injury. International Journal of Neuroscience.

Demaree, H. A., \& Harrison, D. W. (in press-b). A neuropsychological model relating self-awareness to hostility. Neuropsychology Review.

Demaree, H. A., \& Harrison, D. W. (in press-c). Physiological and neuropsychological correlates of hostility. Neuropsychologia.

Edlund, M. J., Swann, A. C., \& Clothier, J. (1987). Patients with panic attacks and abnormal EEG results. American Journal of Psychiatry, 144, 508-509.

Ekman, P., Davidson, R. J., \& Friesen, W. V. (1990). The Duchenne smile: Emotional expression and brain physiology, II. Journal of Personality \& Social Psychology, 58, 342-353.

Ekman, P., Levenson, R. W., \& Friesen, W. V. (1983). Emotions differ in autonomic nervous system activity. Science, 221, 1208-1210.

Folstein, M. F., Maiberger, R., \& MCHugh, P. R. (1977). Mood disorder as a specific complication of stroke. Journal of Neurology, Neurosurgery, \& Psychiatry, 40, 1018-1020.

FuredY, J. J., \& SCHER, H. (1989). The law of initial values: Differentiated testing as an empirical generalization versus enshrinement as a methodological rule. Psychophysiology, 26, 120-121.

GAINOTTI, G. (1972). Studies of the functional organization of the minor hemisphere. International Journal of Mental Health, 1(3), 78-82.

Grafman, J., Vance, S. C., Weingartner, H., Salazar, A. M., \&
AmIN, D. (1986). The effects of lateralized frontal lesions on mood regulation. Brain, 109, 1127-1148.

GReENHOUSE, S. W., \& GeISSER, S. (1959). On methods in the analysis of profile data. Psychometrika, 24, 95-112.

Harrison, D. W., Gorelczenko, P. M., \& CoOK, J. (1990). Sex differences in the functional asymmetry for facial affect perception. International Journal of Neuroscience, 52, 11-16.

Haynes, S. G., Feinleib, M., \& Kannel, W. B. (1980). The relationship of psychosocial factors to coronary heart disease in the Framingham study: III: Eight-year incidence of coronary heart disease in the Western Collaborative Group Study. American Journal of Epidemiology, $111,37-58$.

Haynes, S. G., Levine, S., Scotch, N., \& Kannel, W. B. (1978). The relationship of psychosocial factors to coronary heart disease in the Framingham study. I. Methods and risk factors. American Journal of Epidemiology, 107, 362-383.

Heath, R. G. (1982). Psychosis and epilepsy: Similarities and differences in the anatomic-physiologic substrate. Advances in Biological Psychology, 8, 106-116.

Heilman, K. M., Bowers, D., \& Valenstein, E. (1993). Emotional disorders associated with neurological diseases. In K. M. Heilman \& E. Valenstein (Eds.), Clinical neuropsychology (pp. 461-498). New York: Oxford University Press.

Jenkins, C. D., Rosenman, R. H., \& Friedman, M. (1967). Development of an objective psychological test for the determination of the coronary-prone behavior pattern in employed men. Journal of Chronic Disease, 20, 371-379.

Muranka, M., Lane, J. D., Suarez, E. C., Anderson, N. B., Suzuki, J., \& Williams, R. B. (1988). Stimulus-specific patterns of cardiovascular reactivity in Type A and B subjects: Evidence for enhanced vagal reactivity in Type B. Psychophysiology, 25, 330-338

Robinson, R. G., Kubos, K. L., Stark, L. B., Rao, K., \& Price, T. R. (1983). Mood changes in stroke patients: Relationship to lesion location. Comprehensive Psychiatry, 24, 555-566.

Robinson, R. G., Parikh, M., Lipsey, J. R., Starkstein, S. E., \& Price, T. R. (1993). Pathological laughing and crying following stroke: Validation of a measurement scale and a double blind treatment study American Journal of Psychiatry, 150, 286-293.

Siegman, A. W., Anderson, R., Herbst, J., \& Boyle, S. (1992). Dimensions of anger-hostility and cardiovascular reactivity in provoked and angered men. Journal of Behavioral Medicine, 15, 232-257.

Silberman, E. K., \& Weingartner, H. (1986). Hemispheric lateralization of functions related to emotion. Brain \& Cognition, 5, 322-353.

Starkstein, S. E., Robinson, R. G., \& Price, T. R. (1987). Comparison of cortical and subcortical lesions in the production of poststroke mood disorders. Brain, 110, 1045-1059.

Sturgis, E. T., \& Gramling, S. (1988). Psychophysiological assessment. In A. S. Bellack \& M. Hersen (Eds.), Behavioral assessment (p. 235). New York: Pergamon.

SuAREZ, E. C., \& Williams, R. B. (1989). Situational determinants of cardiovascular and emotional reactivity in high and low hostile men. Psychosomatic Medicine, 51, 404-418.

WILDER, J. (1957). The "law of initial values," a neglected biological law and its significance for research and practice. In S. W. Porges \& M. G. H. Coles (Eds.), Psychophysiology (pp. 38-46). Stroudsburg, PA: Dowden, Hutchingson, \& Ross. (Original work published 193i)

Williams, R. B., Lane, J. D., Kuhn, C. M., Melosh, W., White, A. D., \& SCHANBERG, S. M. (1982). A behavior and elevated physiological and neuroendocrine responses to cognitive tasks. Science, 218 . 483-485.

WOLFF, H. G. (1950). Life situations, emotions, and bodily disease. In M. L. Reymert (Ed.), Feelings and emotions: The Mooseheart Symposium (pp. 284-324). New York: McGraw-Hill.

(Manuscript received March 27, 1996; revision accepted for publication September 16, 1996.) 